\title{
The Rate and Indications of Primary Cesarean Section at Dubai Hospital, Dubai Health Authority, Dubai, UAE
}

\author{
Asma Fahad, Tazeen Makhdoom \\ Dubai Hospital, DHA, Dubai, UAE \\ Email: atiffazari@hotmail.co.uk
}

How to cite this paper: Fahad, A. and Makhdoom, T. (2020) The Rate and Indications of Primary Cesarean Section at Dubai Hospital, Dubai Health Authority, Dubai, UAE. Open Journal of Obstetrics and Gynecology, 10, 626-633.

https://doi.org/10.4236/ojog.2020.1050056

Received: April 12, 2020

Accepted: May 4, 2020

Published: May 7, 2020

Copyright $\odot 2020$ by author(s) and Scientific Research Publishing Inc. This work is licensed under the Creative Commons Attribution International License (CC BY 4.0).

http://creativecommons.org/licenses/by/4.0/

\begin{abstract}
The primary cesarean section rate is defined as the percentage of cesarean deliveries out of all births to women who have not had a previous cesarean delivery. Cesarean section rates steadily increased during the last two decades due to various indications. This is a retrospective study conducted at Dubai Hospital at DHA, Dubai UAE from January 2017 to December 2017, the data were collected using special data collection sheet and analyzed by Statistical package for social sciences (SPSS). The rate of primary cesarean section is $15.4 \%$ with fetal distress being the most common cause primary cesarean section. $66 \%$ of the performed cases were primigravida. $85 \%$ were done as emergency cases. Women aged 36 - 40 years were the common age group among the studied cases. Primary cesarean sections are the driving force behind the overall rate of cesarean sections and cause an increase in morbidity in future pregnancies.
\end{abstract}

\section{Keywords}

Primary Cesarean Section, Fetal Distress, Failure to Progress, Malpresentation

\section{Introduction}

The primary cesarean rate is defined as the percentage of cesarean deliveries out of all births to women who have not had a previous cesarean delivery [1].

Cesarean section rates steadily increased during the 16-year study period (1997-2012) and the distributions of contribution of each indication changed across the years, although the principle contributor remained previous cesarean section. Therefore if one wishes to lower the total cesarean section rate, the ap- 
propriate way would be to reduce the primary section rate by increasing the rate of external cephalic version for breech presentation (and safe breech vaginal delivery technique), tightening the criteria for non-reassuring fetal heart pattern (NRFHRP), liberal use of scalp $\mathrm{pH}$ and labor induction at complete 41 weeks' gestation [2].

The primary cesarean rate increased by $68 \%$ (95\% confidence interval [CI]: 67\%, 69\%) between 1979 (11.0\%) and 2010 (18.5\%). Repeat cesarean deliveries increased by $178 \%$ (95\% CI: 176,179$)$ from $5.2 \%$ in 1979 to $14.4 \%$ in 2010 . Cesarean rates increased with advancing age. Within each period, the primary cesarean delivery rates were higher among older than younger women [3].

The trend of CS delivery rate is significant. The reasons behind this increase are mainly related to the possible easy access to safer types of anesthesia and availability of intensive neonatal care unit, more involvement of juniors in decision making, more incontinence and pelvic repair surgeries, lack of maternal antenatal education, and inconsistency in the antenatal care provided [4].

Changes in obstetrical practices were the major influence on the shifting pattern of primary cesarean rates in the 1990s and the early 2000s. Although age, race/ethnicity, and parity distributions of mothers changed in the 1990s, an examination of cesarean births among lower risk mothers showed that rates of primary cesareans in virtually all of the subgroups analyzed shifted in the same pattern: a decrease between 1991 and 1996 followed by a larger increase between 1996 and 2002. Rates increased over time among older mothers, a significant consideration given the growing number of births in this group [5].

Primary cesarean sections are performed for various indications, the three most common indications were as following, failure to progress, non-reassuring FHR tracing, and fetal malpresentation. Failure to progress was of interest as it strongly affected the cohort of primiparous women at term with a singleton gestation in cephalic presentation. Of all primary cesareans in the study, $45.6 \%$ were performed on primiparous women at term with a singleton fetus in cephalic presentation [1].

One study on the indications of caesarean section, showed large annual increases, including indications such as maternal request, suspected macrosomia, multiple gestation, and preeclampsia. Women's cumulative annualized risk for undergoing a primary cesarean delivery for these indications increased significantly over this time period; however, absolute increases were small. The absolute risks of cesarean delivery for the indications of arrest of dilation and non-reassuring fetal heart tracings remained highest among all indications, and both also increased during the study period [6].

Recent increases in primary cesarean delivery rates were explained by changes in maternal characteristics, specifically by changes in age, parity, pre-pregnancy weight, and weight gain during pregnancy. Changes in obstetric practice (namely, reductions in mid-pelvic forceps use, increases in cesarean delivery for breech presentation, labor induction, epidural anesthesia, and obstetrician delivery) also 
contributed to the increase in primary cesarean deliveries [7].

IVF pregnancies have reported higher CS rates compared to the general obstetric population [8].

Several postulations, such as the "precious baby" effect and the high-risk nature of IVF pregnancies have been advanced to explain the above phenomenon [9]. In a study by Meikle et al., National estimate of elective primary cesarean delivery rate, showed a national estimate of the elective primary cesarean delivery rate with attention to indications using a previously derived methodology. The overall elective primary cesarean delivery rate, that is, primary procedures occurring before labor, was rising. Within the group of procedures with unspecified indications, use of a new code for fetal heart rate abnormalities accounts for the largest shift in coding. Some indications were used increasingly such as malpresentation, antepartum hemorrhage, hypertension and severe hypertension, macrosomia, unengaged head, preterm gestation, and maternal soft tissue disorder were more frequent over the study period. Although there was clear nationwide use of the ICD-9 code for fetal heart rate abnormalities, it was unclear how fetal heart rate abnormality is diagnosed, why there is a rapid rise in the use of this new code, and whether there is a relationship between this new code and maternal-choice cesarean delivery or cesarean delivery for fear of medical liability [10].

Primary cesarean section has a major impact on the rate of cesarean sections and its prevention is vital in reducing the rate of cesarean deliveries.

Prevention of the first cesarean will lower the overall cesarean rate. Therefore, interventions that decrease the diagnosis of dystocia are critically important. Midwifery care practices, such as patience with labor duration, emotional support, and interventions that maintain physical normality (e.g., encouraging ambulation) are likely to lower the incidence of dystocia significantly. Similarly, lowering the incidence of fetal intolerance of labor without increasing the risk of fetal acidemia is possible with the use of IA for low-risk NTSV women. Although many of these practices have been used in out-of-hospital birth settings, they could be feasibly adapted for use in the hospital [11].

We aimed in this study to determine the rate primary Cesarean section and the indications of Cesarean section at Dubai hospital in Dubai health authority, Dubai, UAE.

\section{Materials and Methods}

This is a retrospective descriptive review done at Dubai Hospital the main tertiary care at Dubai Emirates, United Arab Emirates. It has large catchment area involves the Northern Emirates specially for the high-risk cases with bed capacity 625 beds. It is well equipped hospital runs by consultants led teams in all specialties and subspecialties of medicine branches.

The study was conducted from January 2017 until December 2017 during this period 2035 were the registered delivery at the obstetrics and Gynecology.

All cases of primary Cesarean section were studied. Those cases following se- 
lection with restricted definition and criterion of being a case of Cesarean section with no history of repeated cause or previous Cesarean section regardless her parity. The data were collected with specific data collection sheet designed for this purpose. The data were entered and analyzed using Statistical Package for Social Studies (SPSS) version 25 in order to find any statistically significant findings.

The Ethical committee at the medical education department, Dubai Health Authority approved the ethical clearance of this study.

\section{Results}

Among the registered deliveries 2035 out of these 315 underwent primary cesarean section; this makes the rate of primary cesarean section is $15.48 \%$ (Figure 1).

The indication of primary Cesarean section among the studied group varied from fetal distress 101 cases (32\%), malpresentation 63 cases (20\%) and failure to progress 26 cases $(8.2 \%)$ were the three most common indications. Other indications also seen; history of myomectomy, history of 4 th degree perineal tear and history of shoulder dystocia, maternal request in view of precious pregnancy/IVF pregnancy with history of infertility (Figure 2).

The age distribution of the cases came as follows; majority of them 236 cases (75\%), seen in between 36 - 40 years group, 44 cases (14\%) at 31 - 35 years, 16 cases (5\%) in 26 - 30 years group, as well 16 cases $5 \%$ at $21-25$ years and three cases $(1 \%)$ with age of less than 20 years.

Regarding the parity two third of the cases were primigravidas (66\%), 94 cases (30\%) were multiparas while the grandmultipara are 12 cases (4\%).

277 cases (88\%) of the Cesarean sections were done for singleton pregnancies, 31 cases $(10 \%)$ for twin pregnancies and 6 cases $(2 \%)$ in triplets (Figure 3 ).

With respect to types of cesarean section 268 cases (85\%) of the cases were done as emergency Cesarean section while 47 cases (15\%) were planned elective surgery.

\section{Caesarean percentage from total deliveries}

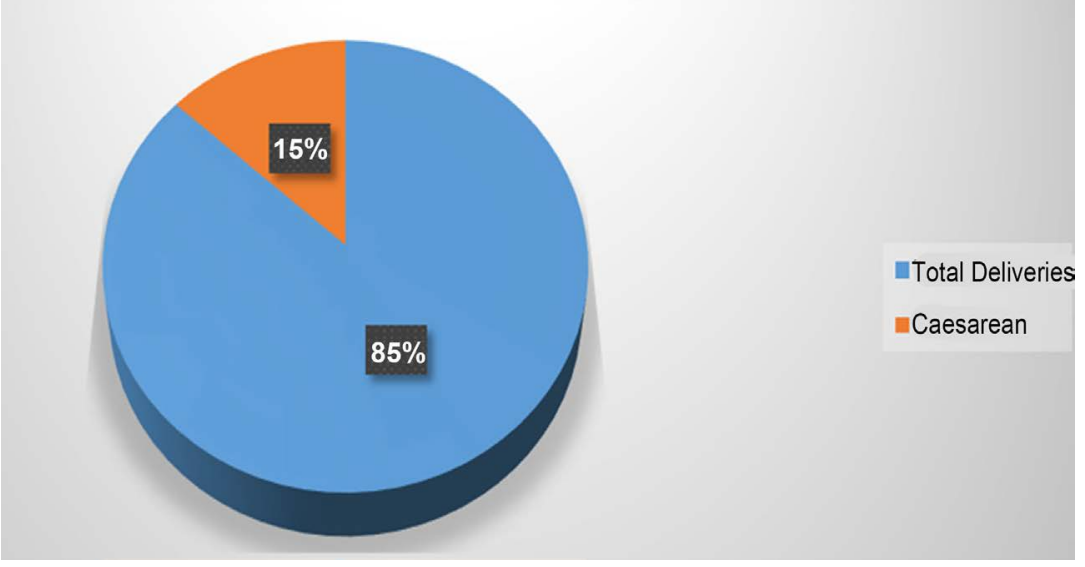

Figure 1 . The rate of primary cesarean section. 


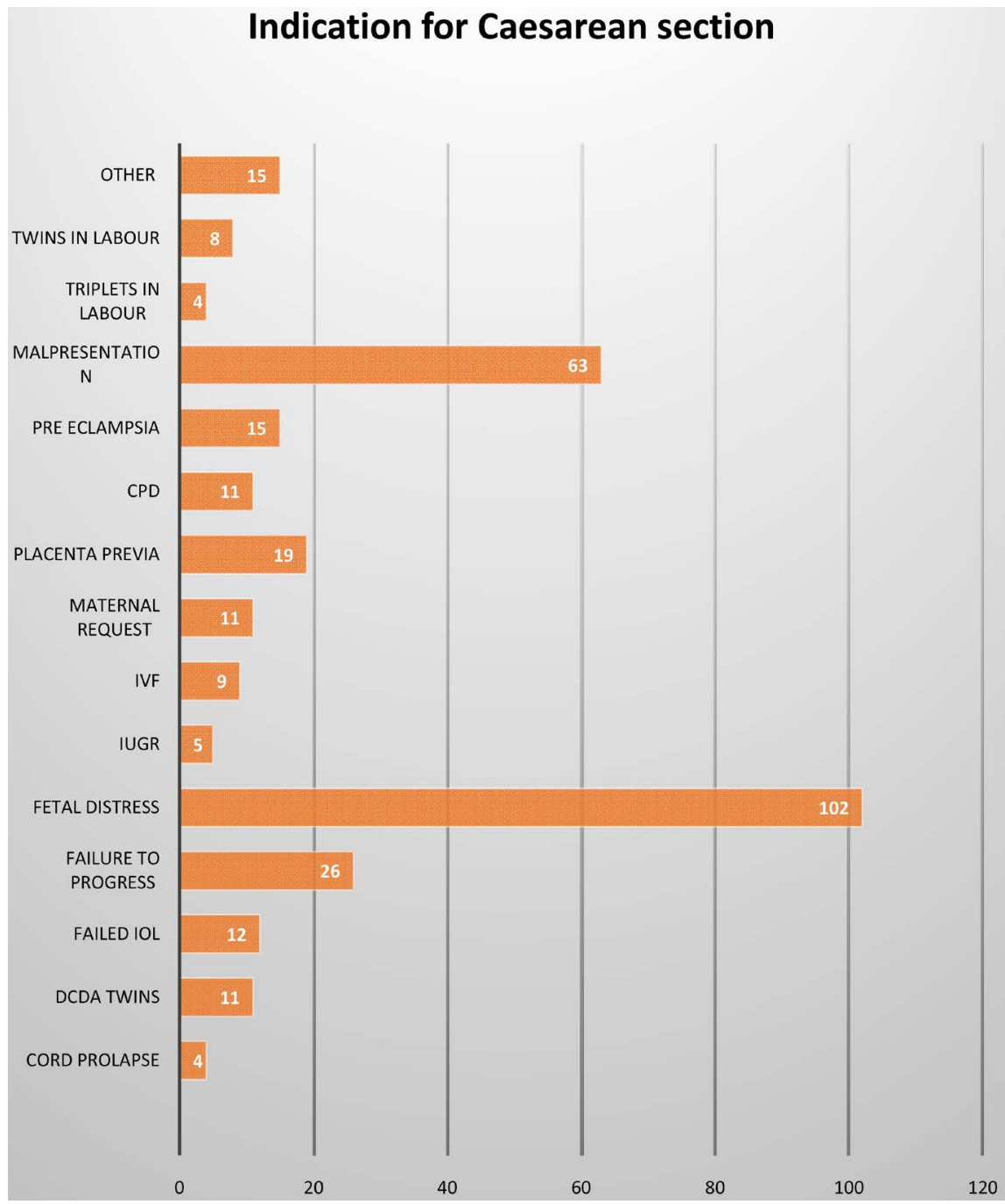

Figure 2. The indications primary cesarean section for cases.

\section{Single Vs Multiple pregnancy}

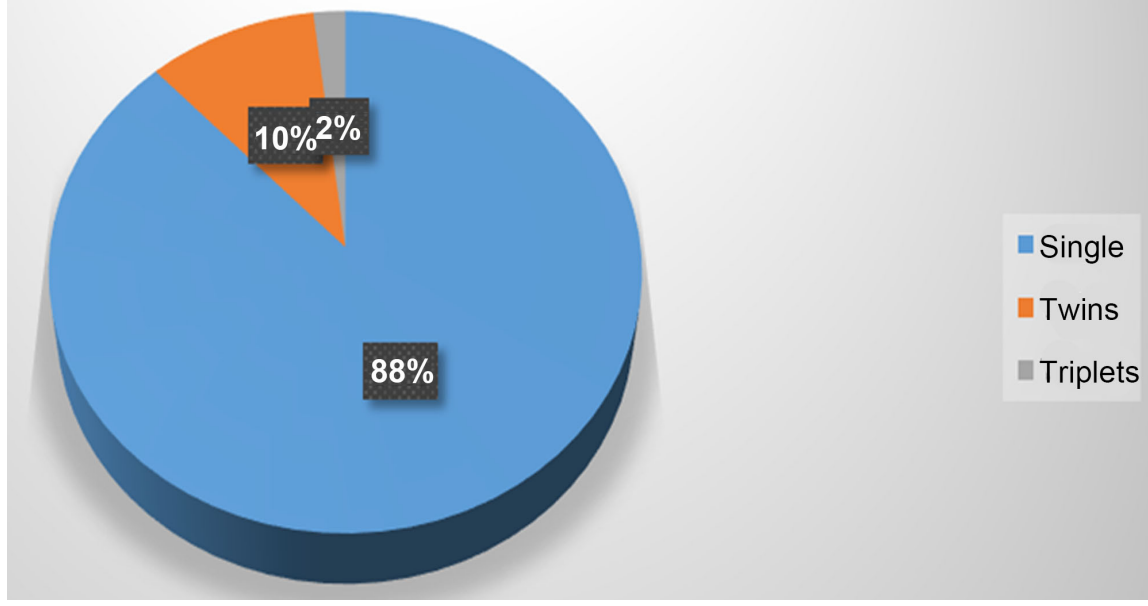

Figure 3. The cases distribution for number of pregnancies. 


\section{Discussion}

In our study the rate of primary cesarean section is $15.4 \%$ which is quite reasonable in comparison with many various rates seen in literature ranging from $23 \%$ and to $60 \%$ in some institute special among the private practice.

The most common indication for primary cesarean section is failure to progress, non-reassuring FHR tracing, and fetal malpresentation [1]. This study proved the same common indications and almost with same order of occurrence and fetal distress is the dominant indication for primary cesarean section.

Our study shows different result concerning the parity where primary cesarean section seen commonly among primigravid almost two third of the case while in study on Primary Cesarean delivery in the United States found that the primary cesarean rate was $30.8 \%$ approximately one third.

We found that most cesarean sections (75\%) were done in women aged 36 40 years of age. A study by Michelle et al. on the maternal age and primary cesarean section rate showed that the cesarean section rates in primiparous women less than 25,25 to 34 , and over 34 years of age were $13.1 \%, 18.5 \%$, and $28.2 \%$, respectively. A similarly dramatic rise with advancing maternal age was seen in multiparous women with rates of $3.4 \%, 4.7 \%$, and $10.1 \%$, respectively, in the three age groups. The strong association between cesarean section and maternal age persisted after multivariate adjustment for induction of labor, epidural anesthesia, meconium-stained amniotic fluid, and fetal distress, and thus these factors do not explain the relationship [12].

Maternal request and precious pregnancy due to IVF is also an evolving indication in primary cesarean sections, as the number of pregnancies due to assisted reproductive techniques increase. A population based study done in Australia of cesarean section after IVF found that the crude rate of cesarean section was 50.1 percent versus 28.9 percent for all other births technology.

Primary cesarean sections are the driving force behind the overall rate of cesarean sections and cause an increase in morbidity in future pregnancies in the form of placental abnormalities such as placenta previa and accreta, risk of uterine rupture and repeat cesarean sections.

\section{Recommendation}

As fetal distress is the major cause of primary cesarean section and a relative term, it should be clearly documented for future audit and research purposes.

The decision should always be taken at a senior level. There should be clear departmental protocols to define fetal distress, computerized analysis of CTG to reduce inter observer error and FBS.

The rate of Cesarean sections can also be reduced by performing ECV for all potential candidates with breech presentation. Staff should be trained and encouraged to conduct breech vaginal delivery.

Universal plotting of partogram, adequate analgesia and good support during labor such as one to one midwifery care will help to reduce the number of pa- 
tients taken for failure to progress.

Cesareans done for maternal request should be booked after senior decision and indications such as IVF pregnancy/precious pregnancy should be discouraged.

Reducing the risk in primary cesarean section will cause a reduction in the overall rate of cesarean section and the associated morbidities.

\section{Limitations}

This is a retrospective hospital-based study in a tertiary health care center and therefore obvious selection bias is unavoidable. In addition, recall bias is a possibility since data was gathered from the medical files of patients some cases might have been documented inaccurately and some cases might have been missed due to incomplete documentation.

\section{Conclusion}

Primary cesarean sections were mainly done in primigravidas with fetal distress being the most common indication. Malpresentation was the second most common cause followed by failure to progress. Trail of ECV, use of computerized CTG to reduce observer error, and use of fetal blood sampling might be able to reduce the rate of primary cesarean sections. Reduction in the primary cesarean section rate is vital to reduce the overall cesarean section rate and as a result decrease in maternal morbidity and mortality.

\section{Conflicts of Interest}

The authors declare no conflicts of interest regarding the publication of this paper.

\section{References}

[1] Boyle, A., Reddy, U.M., Landy, H.J., Huang, C.C., Driggers, R.W. and Laughon, S.K. (2014) Primary Cesarean Delivery in the United States. Obstetric Anesthesia Digest, 34, 150-151. https://doi.org/10.1097/01.aoa.0000452162.16085.a6

[2] Jiang, S. (2004) Difficult Cases in Obstetrics and Gynecology. Chinese Journal of Obstetrics and Gynecology, 6, 410.

[3] Ananth, C.V., Friedman, A.M., Keyes, K.M., Lavery, J.A., Hamilton, A. and Wright, J.D. (2017) Primary and Repeat Cesarean Deliveries: A Population-Based Study in the United States, 1979-2010. Epidemiology (Cambridge, Mass.), 28, 567-574. https://doi.org/10.1097/EDE.0000000000000658

[4] Al-Kadri, H.M., Al-Anazi, S.A. and Tamim, H.M. (2015) Increased Cesarean Section Rate in Central Saudi Arabia: A Change in Practice or Different Maternal Characteristics. International Journal of Women's Health, 7, 685-692. https://doi.org/10.2147/IJWH.S85215

[5] Declercq, E., Menacker, F. and MacDorman, M. (2006) Maternal Risk Profiles and the Primary Cesarean Rate in the United States, 1991-2002. American Journal of Public Health, 96, 867-872. https://doi.org/10.2105/AJPH.2004.052381

[6] Mittal, S., Pardeshi, S., Mayadeo, N. and Mane, J. (2014) Trends in Cesarean Deli- 
very: Rate and Indications. Journal of Obstetrics and Gynecology of India, 64, 251-254. https://doi.org/10.1007/s13224-013-0491-2

[7] Joseph, K.S., Young, D.C., Dodds, L., O’Connell, C.M., Allen, V.M., Chandra, S., et al. (2003) Changes in Maternal Characteristics and Obstetric Practice and Recent Increases in Primary Cesarean Delivery. Obstetrics \& Gynecology, 102, 791-800. https://doi.org/10.1007/s13224-013-0491-2

[8] Reubinoff, B.E., Samueloff, A., Ben-Haim, M., Friedler, S., Schenker, J.G. and Lewin, A. (1997) Is the Obstetric Outcome of in Vitro Fertilized Singleton Gestations Different from Natural Ones? A Controlled Study. Fertility and Sterility, 67, 1077-1083. https://doi.org/10.1016/S0015-0282(97)81442-2

[9] Sullivan, E.A., Chapman, M.G., Wang, Y.A. and Adamson, G.D. (2010) Population-Based Study of Cesarean Section after in Vitro Fertilization in Australia. Birth, 37, 184-191. https://doi.org/10.1111/j.1523-536X.2010.00405.x

[10] Meikle, S.F., Steiner, C.A., Zhang, J. and Lawrence, W.L. (2005) A National Estimate of the Elective Primary Cesarean Delivery Rate. Obstetrics \& Gynecology, 105, 751-756. https://doi.org/10.1097/01.AOG.0000157435.67138.78

[11] Hall, M.J., DeFrances, C.J., Williams, S.N., Golosinskiy, A. and Schwartzman, A. (2010) National Hospital Discharge Survey: 2007 Summary. National Health Statistics Reports, 29, 1-20, 24.

[12] Martel, M., Wacholder, S., Lippman, A., Brohan, J. and Hamilton, E. (1987) Maternal Age and Primary Cesarean Section Rates: A Multivariate Analysis. American Journal of Obstetrics \& Gynecology, 156, 305-308.

https://doi.org/10.1016/0002-9378(87)90273-0 